\title{
Magnetization Control of Magnetic Liquids for Sink-Float Separations
}

\author{
B. Hu ${ }^{*}$, K. van Beek, A. Bosman, P.C. Rem, E.J. Bakker and F. Di Maio \\ Delft University of Technology, Faculty of Civil Engineering and Geosciences, Section Materials and Environment I \\ Recycling Technology, Stevinweg 1, 2628 CN, The Netherlands
}

\begin{abstract}
The cut-density of sink-float separations with water-based magnetic liquids linearly depends on the magnetization of the process liquid. The control of the magnetization of the liquid, by means of measurement followed by extraction of water from the process liquid by membranes or mixing with water or concentrated magnetic liquid, is therefore a critical part of the technology. A potential problem with mixing-in concentrated magnetic liquid is that the process liquid may become inhomogeneous and will segregate in the magnetic field region. Mixing experiments for various scenarios were carried out on a simplified experimental process line and magnetization levels were compared with theoretical models. The results show as process liquid segregation, due to incomplete mixing, can be avoided for all conceivable control strategies by using static mixers. A measurement tool based on a magneto-gravimetric principle was shown to have sufficient sensitivity to detect and control fluctuations of the magnetization of such a fluid in an industrial environment.
\end{abstract}

Keywords: Magnetic density separation, magnetization, magnetic fluid.

\section{INTRODUCTION}

Sink-float separations in magnetic liquids have a considerable potential in recycling because the principle is directly based on the density of the materials, and, unlike most physical separations, does not depend on the size or shape of the separated particles. In a particular system design called Magnetic Density Separation (MDS), the magnetic liquid flows above or beneath a flat magnet and its cutdensity as a separation medium varies strictly in the vertical direction. As a result, the system separates a complex mixture into many different materials in a single process step, using one and the same process liquid. By simply changing the magnetization of the fluid, the MDS can be applied in metal-metal, metal-polymer and polymer-polymer separations [1-3].

In general, the first step of the MDS process is wetting, which is important especially for polymer-polymer applications [4]. If the particles are not properly wetted, air attached to the particles will decrease their effective densities and the separation will be imperfect. If plain water is used for wetting, the introduction of the wet particles into the process liquid will lower its magnetization. After separation, the particles need to be washed with water for some applications or because the magnetic liquid is too expensive to lose with the particles. The rinsing water can be concentrated again by means of a membrane [5] and the concentrate is then recycled into the process liquid. As a result of wetting and washing, the magnetization of the process liquid may become off-spec so that the cut-point of the separation process is affected. For this reason, the quality of the liquid is checked by a magnetization measurement

*Address correspondence to this author at the Department of Civil Engineering and Geosciences, Delft University of Technology, Delft, The Netherlands; Tel: +31-15-27 82897; Fax: +31-15-27 88162;

E-mail: Bin.Hu@tudelft.nl tool and controlled by adding either water or concentrated magnetic liquid.

There are several options to correct the quality of the liquid in industrial processes. If the liquid drifts away from the spec relatively slowly, the quality can be corrected once per day by adding some amount of water or concentrated magnetic liquid during start-up. This strategy has a disadvantage that the added liquid may distribute in an inhomogeneous way over the process liquid and create variations of the magnetization at the scale of the system as a whole or across the height of the separation channel, as a result of segregation of ill-mixed liquid. The first problem can be avoided by adding the correction liquid to a large well-stirred tank that is usually part of the system. The second problem can only be solved by intensive on-line mixing. The alternative process control option is to correct the process liquid on-line, continuously. In this latter case, less mixing energy is needed to avoid segregation. In this paper, we study three mixing options on a simplified process line and report theoretical and experimental results on the mixing behavior. In order to keep track of fluctuations of the magnetization in an industrial process, a magnetization measurement tool was developed based on a magnetogravimetric principle and tested for sensitivity in the laboratory and in an industrial environment.

\section{THEORETICAL BACKGROUND}

Water-based magnetic fluids have a relatively low density $\rho_{\text {magf }}$, comparable to that of water, but in a gradient magnetic field $B$ the weight of the fluid may be artificially increased or decreased, because the force on the fluid is the sum of gravity and the magnetic force. By a clever arrangement of the magnetic induction $B$, it is possible to create an apparent density $\rho_{\text {app }}$ of the medium which varies only with the vertical coordinate $z$. If the fluid has a magnetization $M$, the apparent medium density varies exponentially with $z$ (Eq. 1): 
$\rho_{\text {app }}=\rho_{\text {magf }}+\frac{2 \pi M B_{0}}{g w} e^{-2 \pi z / w}$

Here $w$ is the wavelength of the magnetic field and $g$ is the acceleration of gravity.

Fig. (1) shows an example of the apparent density of a process fluid in the separation channel before and after correction by adding concentrated magnetic liquid. The example shows the effect of correcting the magnetization from an original $900 \mathrm{~A} / \mathrm{m}$ to an average of $1000 \mathrm{~A} / \mathrm{m}$ by dispersing $1 \mathrm{vol} \%$ of concentrate. The resulting variations of density were calculated for magnetic field amplitude of $0.6 \mathrm{~T}$ and a wavelength of $240 \mathrm{~mm}$. If the concentrate is not wellmixed and bodies of concentrated liquid are large enough to segregate towards the surface of the magnet, the magnetization will be off-spec in both the top and bottom part of the channel.

One factor affecting the size of bodies of concentrated liquid in the process fluid is diffusion. The diffusion of magnetite nano-particles in a magnetic fluid can be determined by the Einstein diffusion equation [6] (Eq. 2):

$$
S^{2}=2 D \tau
$$

in which $\tau$ is the elapsed time, $S$ is the diffusion length scale, and $D$ is the diffusion coefficient of the magnetite particle in water given by the Maxwell-Stefan equation [7]:

$$
D=\frac{R T}{L 3 \pi \eta d_{\text {nano }}}
$$

Here $R$ is the gas constant, $T$ is the absolute temperature, $L$ is Avogadro's number, $\eta$ is the dynamic viscosity of the magnetic fluid and $d_{\text {nano }}$ is the diameter of the particles suspended in the fluid.

The diffusion coefficient determined by (Eq. 3) is about $10^{-10} \mathrm{~m}^{2} / \mathrm{s}$. If the time between injection of the water or concentrated liquid and the arrival of the process fluid in the separation channel is 3-4 seconds, the diffusion scale is approximately $25 \mu \mathrm{m}$ (Fig. 2), which means that bodies of water or concentrate with a size substantially smaller than 25 $\mu \mathrm{m}$ will have effectively diffused into the surrounding process fluid before they get to the magnetic field. Larger droplets, which exceed the critical diffusion size, may survive to the separation channel and cause segregation of the process fluid.

A second issue is whether droplets bigger than the critical diffusion size will actually segregate in the magnetic field. Any droplet of water or concentrated magnetic fluid which does not diffuse into the process liquid before reaching the magnetic field behaves as a particle with a density lower or higher than the apparent density of the process liquid, and so it will float or sink in the separation channel.

A small droplet of diameter $d$, volume $V_{\text {droplet }}$ and apparent density $\rho_{\text {droplet }}$ immersed in a magnetic fluid of apparent density $\rho_{\text {app }}$ and dynamic viscosity $\eta$ will experience a gravity force $\left(F_{\mathrm{G}}\right)$, a drag force $\left(F_{\mathrm{D}}\right)$ and a buoyancy force $\left(F_{\mathrm{B}}\right)[8]($ Eq. 4-6):

Gravity force: $F_{G}=\rho_{\text {droplet }} \cdot V_{\text {droplet }} \cdot g$

Eq. 4

Drag force: $F_{D}=3 \pi \eta d v_{t}$

Eq. 5

Buoyancy force: $F_{B}=\rho_{a p p} \cdot V_{\text {droplet }} \cdot g$

Eq. 6

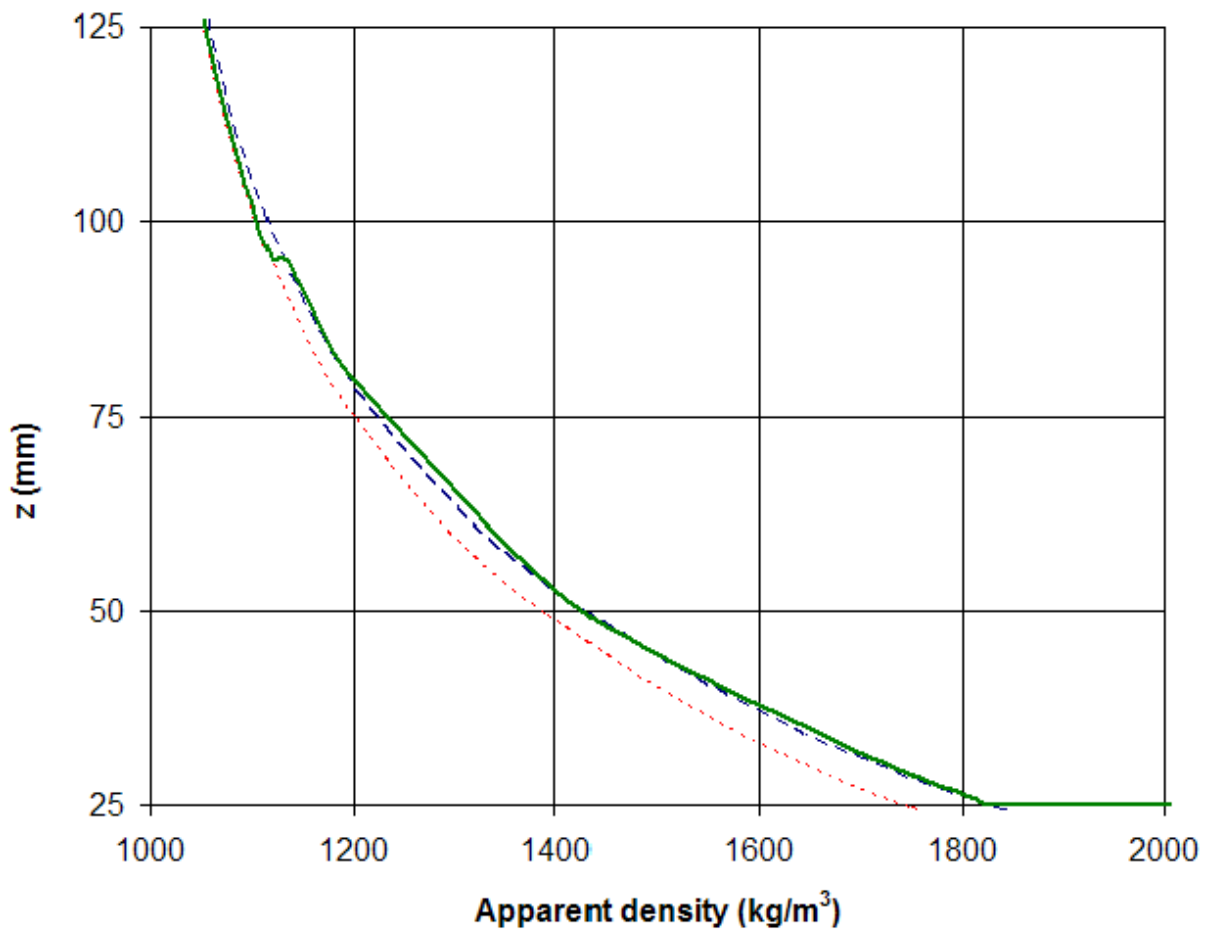

Before correction - - - -Homogeneous —_ Segregated

Fig. (1). Effect of segregation on the apparent density of the process liquid in the separation channel. 


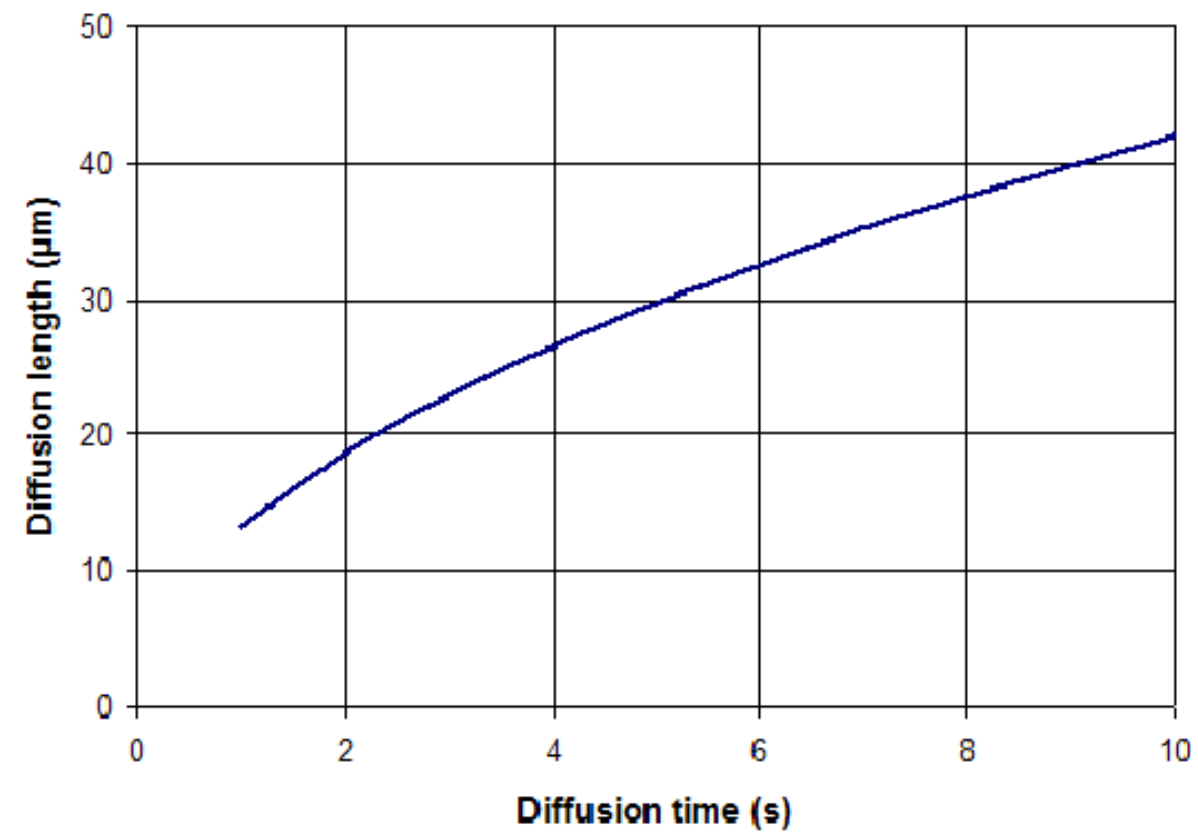

Fig. (2). Diffusion distance of magnetic fluid as a function of elapse time.

Therefore, the settling velocity of the droplets, $v_{\mathrm{t}}$, is given by (Fig. 3) (Eq. 7):

$v_{t}=\frac{\left(\rho_{\text {droplet }}-\rho_{\text {magf }}\right) d^{2} g}{18 \eta}$
In the simulation shown in (Fig. 3), water droplets of diameter $200 \mu \mathrm{m}$ fed into the magnetic field from different heights float upward in the process liquid relatively slowly. The concentrated magnetic fluid droplets are attracted by the magnet and produce a flow of concentrated magnetic fluid (apparent density $2260 \mathrm{~kg} / \mathrm{m}^{3}$ ) on the bottom of the

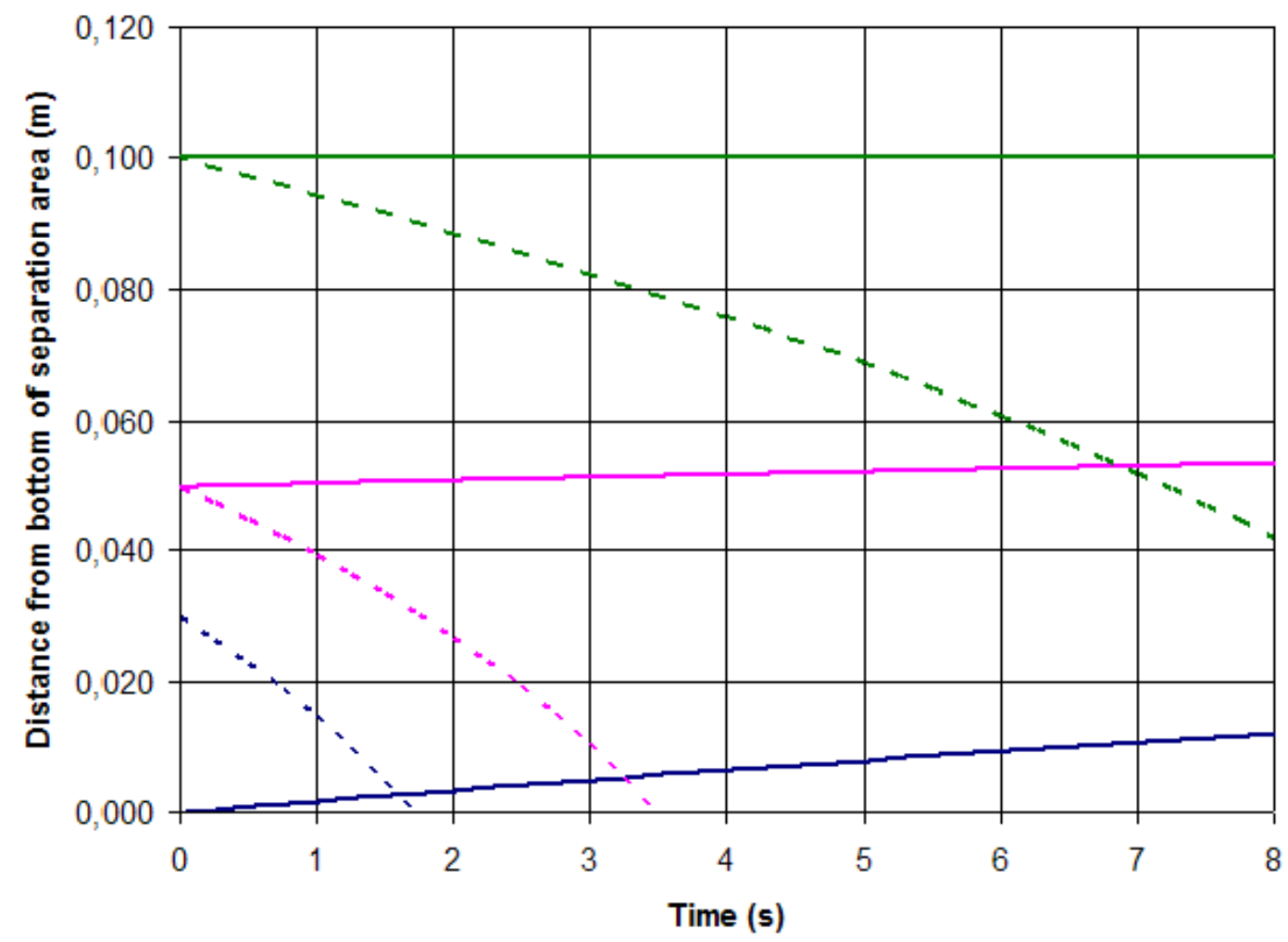

Fig. (3). Simulated trajectories of droplets of water and concentrated magnetic fluid $(\mathrm{d}=200 \mu \mathrm{m})$. The magnetization of the process liquid is $900 \mathrm{~A} / \mathrm{m}$. The straight lines are water droplets; the dashed lines are for concentrated magnetic fluid droplets $(12000 \mathrm{~A} / \mathrm{m})$. 


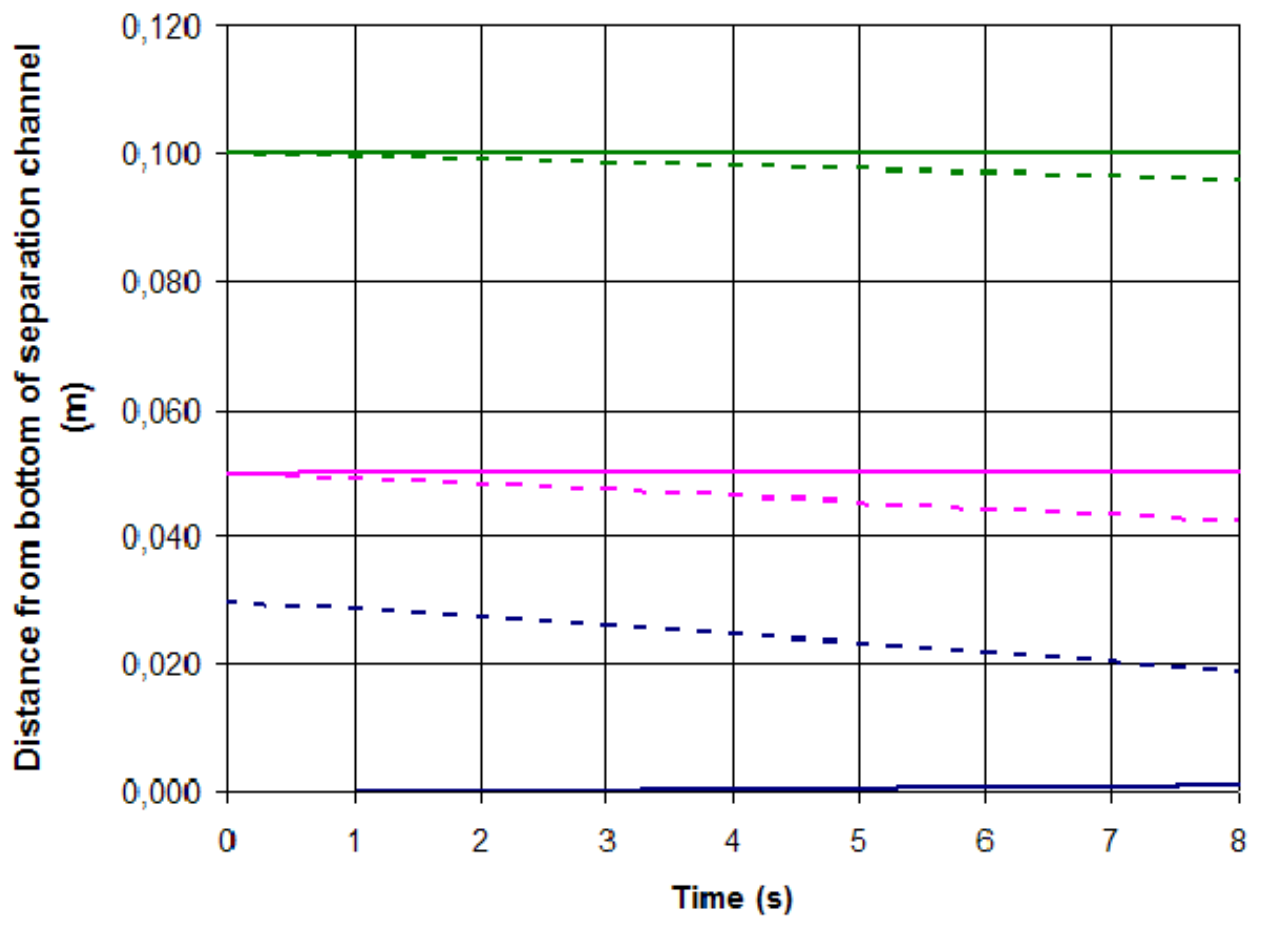

Fig. (4). Simulated trajectories of water and concentrated magnetic fluid droplets $(d=60 \mu \mathrm{m})$.

separation channel within several seconds. It is even possible that the magnetic fluid will remain on the bottom of the separation channel, due to the attraction by the field, if the magnet is sufficiently strong. As a result, the apparent densities of the magnetic fluid in the magnetic field become lighter and heavier on the top and bottom, respectively.

The distribution is significantly improved by reducing the sizes of droplets. In (Fig. 4), droplets of both water and concentrated magnetic fluids $(\mathrm{d}=60 \mu \mathrm{m})$ distribute homogeneously in the magnetic field. There is a slight settling for the magnetic fluid droplets, and the settling distance is smaller than $1 \mathrm{~cm}$ in 8 seconds. Taking into consideration that the height of each layer in sampling are is $2 \mathrm{~cm}$, the settling distance is acceptable to avoid segregation. Hence, any water droplet smaller than $60 \mu \mathrm{m}$ will not create segregation of the process liquid. As a result, the liquid does not segregate and the separation will be not influenced.
Based on the theory presented above, the distribution of droplets essentially depends on the sizes of droplets of water or concentrated magnetic fluid, which on the other hand may cause segregation. By introducing mixing, it is possible to reduce the sizes of the droplets and avoid segregation.

\section{EXPERIMENTAL METHODS}

\subsection{MDS and Mixing}

The experimental setup was made of feeding zone, mixing area, separation channel and sampling area as shown in (Fig. 5). The flow rate was $8.6 \mathrm{~m}^{3} / \mathrm{h}$. About $1 \mathrm{~kg}$ of water or concentrated magnetic fluid $(12000 \mathrm{~A} / \mathrm{m})$ was pumped into the feeding zone in approximately 1 minute for each single experiment. Two types of mixers were tested: a static mixer and an impeller mixer (Fig. 6). The flow speed in the separation area was $0.08 \mathrm{~m} / \mathrm{s}$ and the liquid head was $0.1 \mathrm{~m}$. The magnetizations of the process liquids ranged from 800 to $1000 \mathrm{~A} / \mathrm{m}$. In this situation, $1 \mathrm{~kg}$ of concentrated magnetic

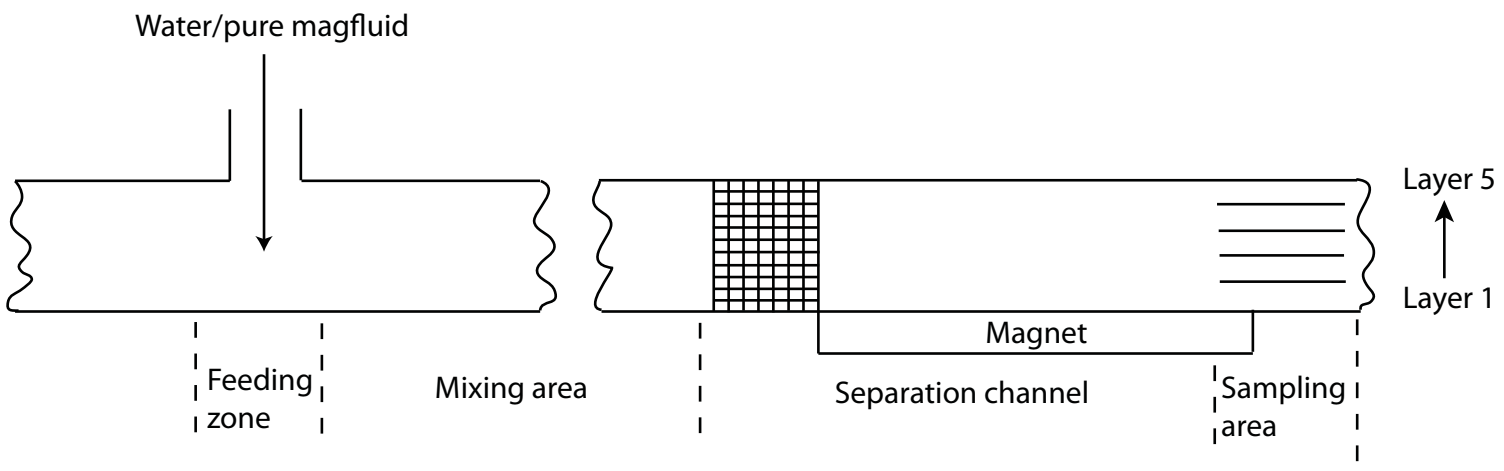

Fig. (5). Experimental setup. 
fluid injected in the system produced approximately $10 \%$ increase of the magnetization in each layer of the separation channel. The pressure drop across the static mixer was 9 mbar. The speed of the impeller mixer was 300 RPM. The sampling started after 30 to 45 seconds from the injection of the water or concentrated magnetic fluid to the system. Five samples were taken from each layer in the sampling area and their magnetization was analyzed. Apart from the tests with the two types of mixers, one experiment was done without mixing as a reference.

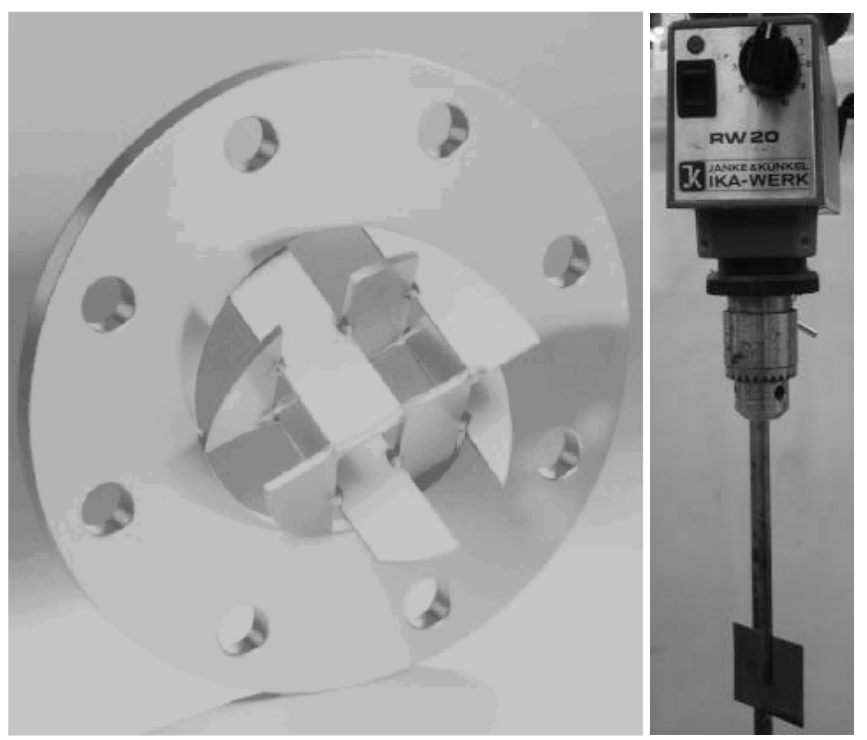

Fig. (6). Static mixer DN 65 and Impeller mixer.

\subsection{Nano-Filtration}

A nano-filtration is utilized to emulate the online control of the concentration of the process liquid. After separation, the process liquid sticking on the particles is reclaimed so that water and concentrated magnetic fluid are produced continuously by means of a nano-filtration membrane. The water may be used for wetting and finally it is continuously introduced into the process liquid with particles. Meanwhile the concentrate may be added into the system to compensate the water which is used for wetting. In this way, water or concentrated magnetic fluid is probably accumulated in the long run if the mixing unit does not work as expected.

The pilot nano-filtration unit used in the experiment consisted of a spiral wound nano-filtration element with a 62 $\mathrm{mm}$ diameter. A $\mathrm{TFC}^{\odot}$ polyamide membrane with a surface of $1.1 \mathrm{~m}^{2}$ was used to treat the process liquid (shown in Fig. 7). During the test, the membrane produced $24 \mathrm{l} / \mathrm{h}$ of water with a total feed of $250 \mathrm{l} / \mathrm{h}$. The test was performed in a closed loop: the concentrate (magnetic fluid) and permeate (water) produced by the membrane were fed to the feeding zone simultaneously, and mixed with the process liquid by the static mixer. After sampling, the main stream left in each layer were pumped out and finally mixed in a storage vessel, from which a small amount of magnetic fluid was extracted and fed into the nano-filtration device. The system was running for more than 70 minutes. Samples were taken at the $15^{\text {th }}$ and $70^{\text {th }}$ minute in order to find out whether there was accumulation of water or concentrated magnetic fluid in the long run.

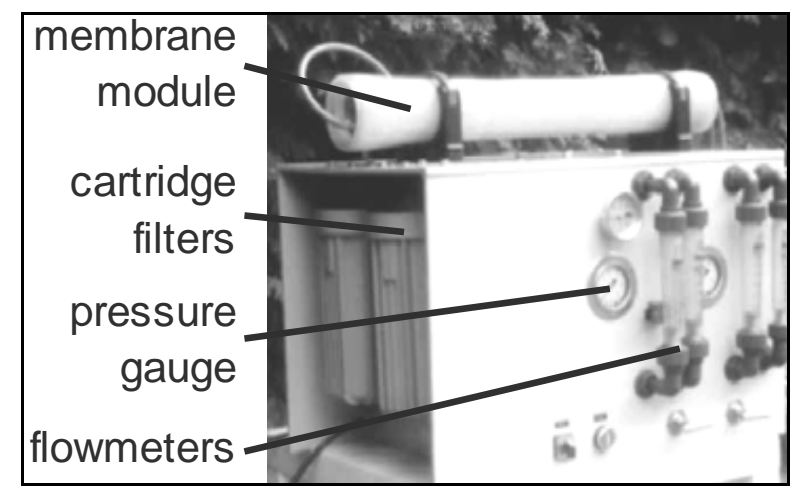

Fig. (7). Nano-filtration test setup.

\subsection{Magnetization Measurement Tool}

All the samples from the tests were analyzed by using a simple setup (Fig. 8). A cup filled with magnetic fluid of certain volume $(V)$ is placed in the magnetic field $(B)$. The distance between the cup and the magnet is $h$. The magnetic force $\left(F_{\mathrm{mag}}\right)$ acting on the $V$ is measured by means of the balance. The magnetization $(M)$ is determined by (Eq. 8):

$$
M=\frac{F_{m a g}}{V \cdot \nabla B}
$$

Based on the simplified magnetization measurement setup described above, a magnetization measurement tool was developed at TU Delft. The advantage of the tool is continuous measurement of the magnetization of the liquid. The output of the tool can be transferred to a PLC (programmable logic controller), which is a common system unit to control industrial processes. The magnetization measurement tool can control both the reclamation process with nano-filtration and the quality of the process liquid. It is necessary to know the accuracy of the magnetization measurement tool for both duties.

Five different dilutions of concentrated magnetic liquid were made: a dilution of 10, 20, 50, 100 and 200. These dilutions were measured with the tool. Because of the inaccuracy of the dilutions itself, a second method was used to measure the magnetization of the liquid; atomic absorption spectroscopy (AAS) (a Varian AA640 was used). This technique can determine the concentration of a particular metal element in a sample, in this case Fe.

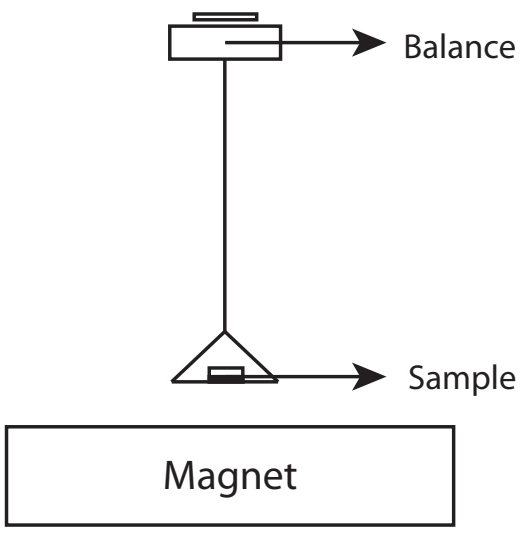

Fig. (8). Magnetization measurement setup. 


\section{RESULTS and DISCUSSION}

\subsection{MDS and Mixing}

The tests were performed by adding water to the system (Fig. 5) first. It was found that the magnetization of the magnetic fluid did not appreciably vary after $1 \mathrm{~kg}$ water was fed within 1 minute (Fig. 9). The differences of the magnetizations in Layer 2 to 4 were generally less than $1 \%$, when no mixer or the static mixer was applied. However, the magnetization in Layer 5 decreased of $2 \%$. The liquids in all layers were diluted by water when the impeller mixer was used. However, there was a sampling mistake in Layer 3 which caused a variation of nearly $8 \%$ in magnetization before and after water was introduced. Another issue which may have introduced error is the inaccuracy of the magnetization measurement itself. According to calculations, on average only $0.8 \%$ of the magnetization difference is able to be created by $1 \mathrm{~kg} / \mathrm{min}$ water in each layer. But the sensitivity of the instrument was not sufficient to measure such small differences. Consequently, there is no enough information to estimate the sizes of the added droplets of water in the separation channel.

In the second experiment, concentrated magnetic fluid was fed to the system instead. The corresponding variations of the magnetization of the process liquid in each layer were compared. As shown in Fig. (10), without extra mixing, the magnetization of the liquid in Layer 2 to 4 increased $10 \%$ while the magnetization in layers 1 and 5 was higher and lower respectively. The reason is that the without mixer, the added droplets of concentrated magnetic fluid were too big to be uniformly distributed when in the separation channel. Therefore, because of the combined effect of both the gravity and magnetic fields, they settled to the bottom of the separation channel so that the magnetization in layer 1 was increase. On the contrary, the flow in the top layer (Layer 5) became less magnetic. This test demonstrates that within the diffusion time (3-4 seconds) both the concentration and the size of the droplets are above the minimal values which cause segregation. Moreover, according to the previous simulation results, the size of the droplets was larger than 60 $\mu \mathrm{m}$ which is the upper limit for avoiding segregation on theoretical ground.

By using the static mixer, the measured increase of the magnetization of the magnetic fluid was $10 \%$ in each layer. This means that the added concentrated magnetic fluid has been uniformly distributed in the process fluid due to the action of the static mixer, which broke down the droplets of magnetic fluid to a size less than $60 \mu \mathrm{m}$. Calculation showed that the power dissipated by the static mixer was $14 \mathrm{~W} / \mathrm{m}^{3}$, while the one of the impeller mixer was only $0.6 \mathrm{~W} / \mathrm{m}^{3}$ which was not enough to break down the droplets. The latter explains the in-homogeneity of the magnetic fluid across the separation channel when the impeller mixer was used.

It is concluded that the process liquid may be mixed inhomogeneous in the separation channel if a big amount of water or concentrated magnetic fluid is injected into the system in a short time. The homogeneity can be increased by using a static mixer.

\subsection{Nano-Filtration}

The experiment layout described above shows that the magnetization of the process liquid was well controlled through the simultaneous operation of both the nanofiltration membrane and the static mixer. Therefore, there was no variation of magnetization in each layer following the combined injection of both water and reclaimed magnetic fluid to the system. It implies that neither water nor concentrated magnetic fluid was accumulated within 70 minutes of continuous operation of the system. Thus the static mixer has been proved to work effectively even in a long run.

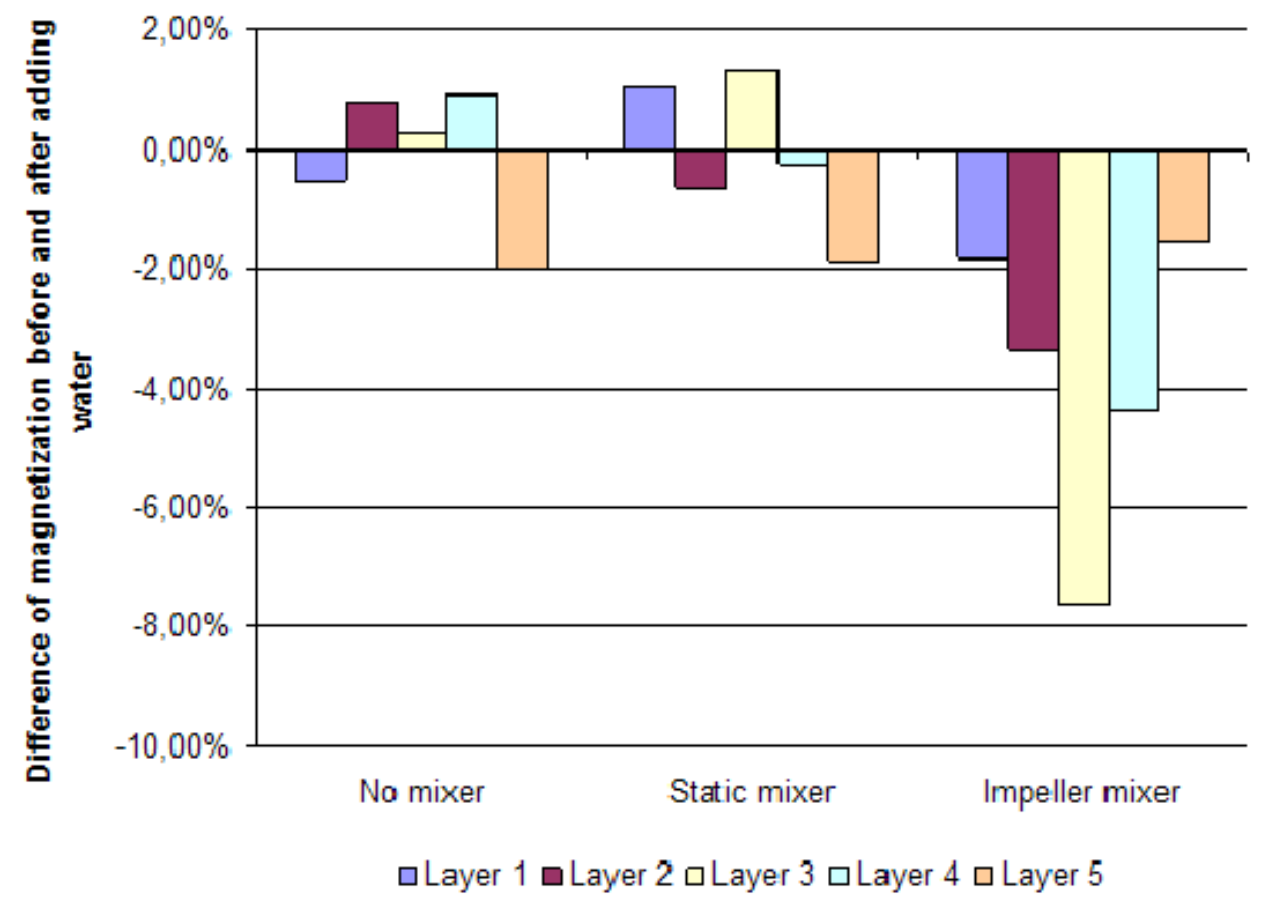

Fig. (9). Magnetization differences of the magnetic fluid in various layers before and after adding water by using different mixers. 


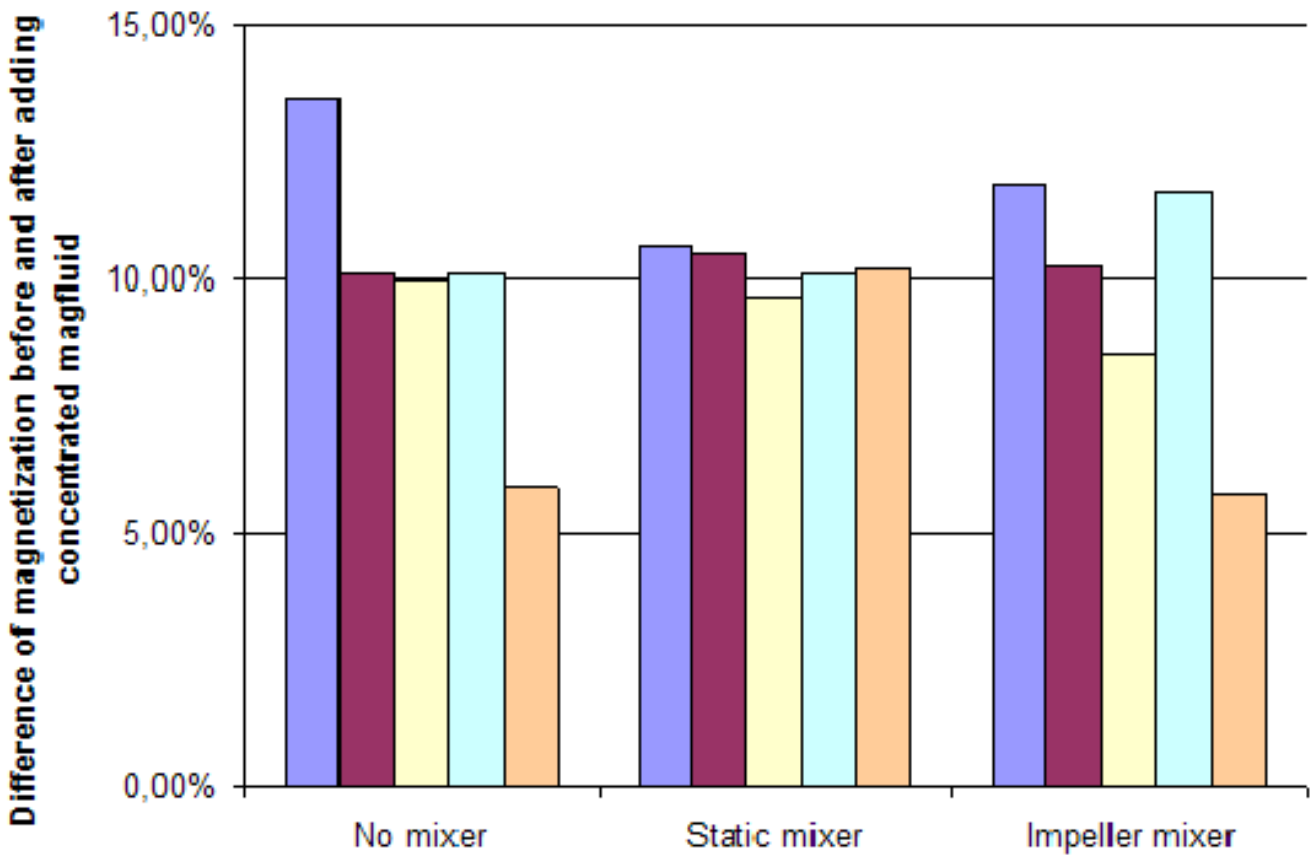

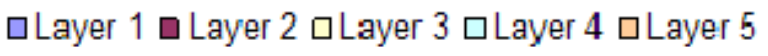

Fig. (10). Magnetization differences of the magnetic fluid in various layers before and after adding concentrated magnetic fluid.

\subsection{Magnetization Measurement Tool}

An electronic magnetization measurement tool (MMT) was designed along the principle of Fig. (8). This tool was calibrated and then tested for sensitivity in laboratory and industry environment. The magnetic liquid as received from the supplier was diluted 10, 20, 50, 100 and 200 times. This was done twice. The content of $\mathrm{Fe}$ (ppm) was measured using AAS. The results are shown in Table 1. The diluted liquid samples of the second AAS measurement were tested with the MMT. Because of the continuous measurement with MMT, the output is varying in time. The lowest and highest output for the specific samples is given in Table 2 .

The results of Table $\mathbf{1}$ show that it is difficult to make diluted samples to an accuracy of better than $3 \%$ in final

Table 1. Results of the Two AAS Measurements Compared to Dilution Factor of the Magnetic Liquid

\begin{tabular}{|c|c|c|c|c|c|}
\hline Dilution Factor & $\begin{array}{c}1^{\text {st }} \text { AAS Measurement } \\
(\text { ppm Fe) }\end{array}$ & $2^{\text {nd }}$ AAS Measurement (ppm Fe) & Average (ppm Fe) & StDev* (ppm Fe) & StDev*/Average (\%) \\
\hline 10 & 22,962 & 21,992 & 22,477 & 686 & 3.05 \\
\hline 20 & 11,499 & 11,393 & 11,446 & 75 & 0.65 \\
\hline 50 & 4,787 & 4,295 & 4,541 & 348 & 7.66 \\
\hline 100 & 2,473 & 2,322 & 2,398 & 107 & 4.45 \\
\hline 200 & 1,233 & 1,205 & 1,219 & 20 & 1.62 \\
\hline
\end{tabular}

*StDev: Standard deviation.

Table 2. Results of the MMT Compared to the Second AAS Measurement

\begin{tabular}{|c|c|c|c|c|c|}
\hline $2^{\text {nd }}$ AAS Measurement (ppm Fe) & MMT Lowest & MMT Highest & Average & StDev* & Stdev* (\%) \\
\hline \hline 21,992 & 6,585 & 6,605 & $6,595.0$ & 14.1 & 0.21 \\
\hline 11,393 & 3,160 & 3,169 & $3,164.5$ & 6.4 & 0.20 \\
\hline 4,295 & 1,096 & 1,100 & $1,098.0$ & 2.8 & 0.26 \\
\hline 2,322 & 560 & 562 & 561.0 & 1.4 & 0.25 \\
\hline 1,205 & 283 & 284 & 283.5 & 0.7 & 0.25 \\
\hline
\end{tabular}




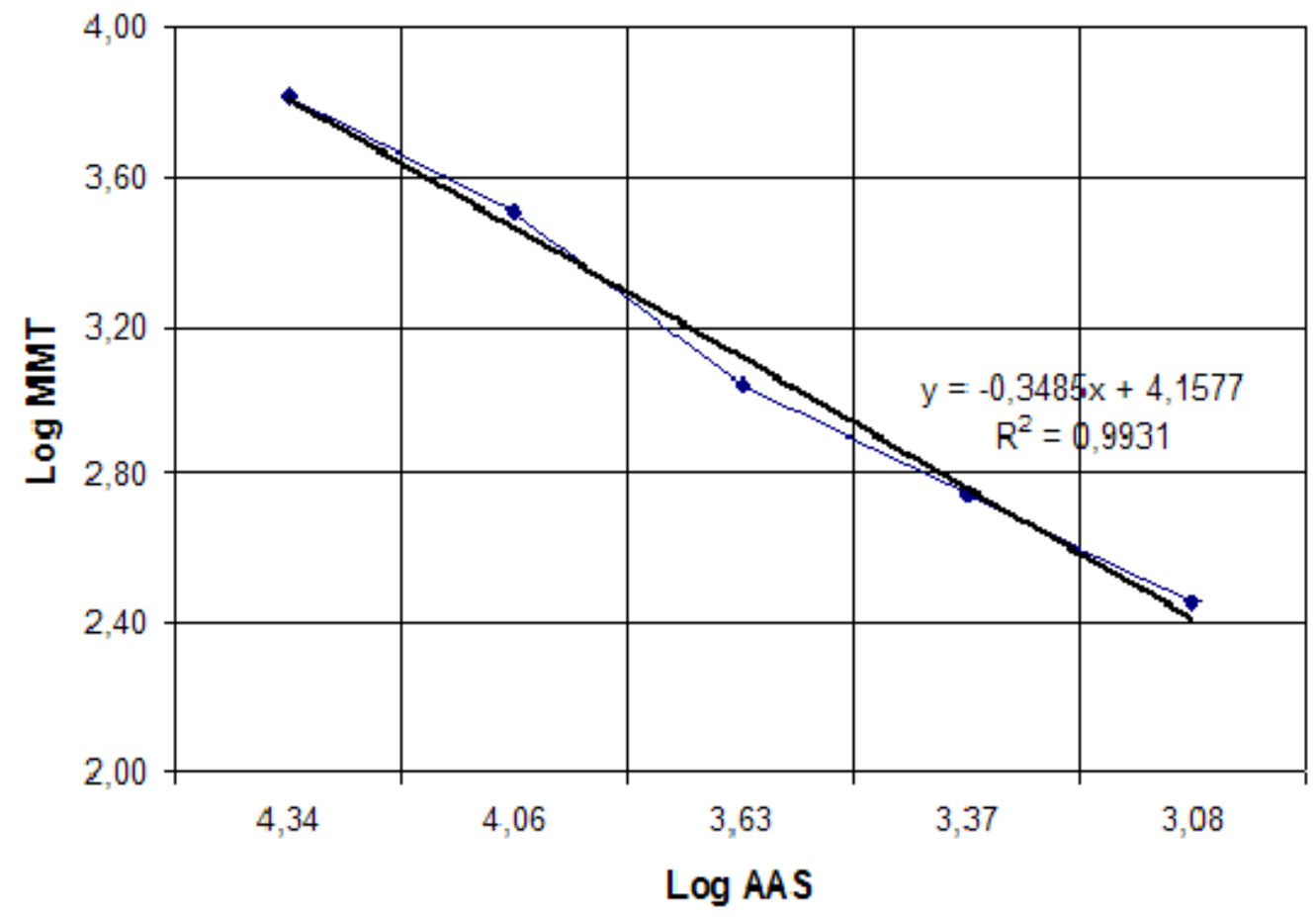

Fig. (11). Comparison of the measurements with AAS and MMT.

concentration. The two samples with dilution factor 50 have a standard deviation of more than $7 \%$ of the average. This is because the diluted samples are made manually. This makes clear why just testing the MMT with diluted samples is not an accurate method. It also shows that making quantities of magnetic liquid with a certain magnetization for MDS applications in the laboratory need checking of the magnetization.

The results of Table $\mathbf{2}$ show that the variation in output in time of the MMT is not a significant issue for separation with MDS in industry and also that the sensitivity of the tool is quite adequate. The average of the MMT output is compared with the second AAS measurement results and can be found in Fig. (11). This shows that the MMT, if properly calibrated, can not give a precise absolute magnetization of the liquid, but it is relatively accurate enough. By continuously measuring the magnetization of the process liquid, the MMT can be used to monitor any significant change in magnetization, when this change is more than $0.5 \%$. This is sufficiently accurate for industrial purposes.

\section{CONCLUSIONS}

The cut-density of sink-float separations in magnetic liquids depends linearly on the magnetization of the process liquid. The control of the magnetization of the liquid is therefore a critical part of the technology. There are several options to correct the quality of the liquid in industrial processes. However, a potential problem is that the process liquid may become inhomogeneous and will segregate in the magnetic field region. In this paper, three mixing options on a simplified process line were studied and theoretical and experimental results on the mixing behavior were reported. There are two parameters governing the distribution of the droplets in the process liquid: the size of the droplets and the value of the magnetic field. For a magnetic field amplitude of $0.6 \mathrm{~T}$ and a wavelength of $240 \mathrm{~mm}$, the critical size of the droplets of concentrated magnetic liquid $(12000 \mathrm{~A} / \mathrm{m})$ is 60 $\mu \mathrm{m}$. The experiments show that segregation of the process liquid due to incomplete mixing can be avoided by utilizing a static mixer which is able to reduce the size of droplets of concentrated magnetic fluid down to $60 \mu \mathrm{m}$. However, there is no enough information to estimate the sizes of the added droplets of water in the separation channel. By combining a nano-filtration membrane and a static mixer, process liquid is properly controlled online as well. Based on a magnetogravimetric principle, a magnetization measurement tool was designed and shown to have sufficient sensitivity to detect and control fluctuations of the magnetization of the process liquid in an industrial environment.

\section{ACKNOWLEDGEMENTS}

The study was developed with thanks to the financial support of the European Commission in the framework of the FP7 Collaborative project "Magnetic Sorting and Ultrasound Sensor Technologies for Production of High Purity Secondary Polyolefins from Waste (W2Plastics)", Grant Agreement No. 212782.

\section{REFERENCES}

[1] E. J. Bakker and P. C. Rem, "Magneto-hydrostatic separation of PET", Proceedings of the 5th Int. Conference for Conveying and Handling of Particulate Solids, 2006.

[2] E. J. Bakker, P. C. Rem, and N. Fraunholcz, "Upgrading mixed polylefin waste with magnetic density separation", Waste Management, vol. 29, pp. 1712-1717, May 2009.

[3] L. Muchova, E. Bakker, and P. Rem, "Precious metals in municipal solid waste incineration bottom ash", Water, Air, and Soil Pollution: Focus, vol. 9, pp. 107-116, April 2009.

[4] E. J. Bakker, P. C. Rem, and D. Hartmann, "Magnetic density separation", Int. Conf. on Envirorunental Science and Technology 2007, American Academy of Sciences, 2007. 
[5] H. Agterhuis, D. Trambitas, E. J. Bakker, and P. C. Rem, "Comparing methods for reclaiming ferrofluids used in materials separation", in the 22nd international conference on solid waste technology and management, Philadelphia, PA, USA, 2007.

[6] M. A. Islam, "Einstein-Smoluchowski diffusion equation: A discussion", Physica Scripta, vol. 70, pp. 120-125, February 2004.
[7] J. A. Wesselingh and R. Krishna, Mass Transfer. Chichester: Ellis Horwood Limited, 1990.

[8] V. Murariu, J. Svoboda, and P. Sergeant, "The modelling of the separation process in a ferrohydrostatic separator", Minerals Engineering, vol. 18, pp. 449-457, April 2005.

(C) Hu et al.; Licensee Bentham Open.

This is an open access article licensed under the terms of the Creative Commons Attribution Non-Commercial License (http://creativecommons.org/licenses/by$\mathrm{nc} / 3.0 /$ ) which permits unrestricted, non-commercial use, distribution and reproduction in any medium, provided the work is properly cited. 